\title{
DUAL VARIATIONAL PRINCIPLES FOR DIFFUSION EQUATIONS*
}

\author{
$\mathrm{BY}$ \\ ISMAEL HERRERA ${ }^{1}$ AND JACOBO BIELAK ${ }^{2}$ \\ Universidad Nacional Autonoma de México, México, D.F.
}

1. Introduction. The results presented in this paper were obtained in the course of an investigation on transient flow in porous media, but they are equally applicable to other problems governed by the heat diffusion equation.

Variational principles have been used extensively in ground water hydrology. Recently, they have served as basis for the development of finite-element techniques for fluid flow problems in porous media [1,2].

A variational principle is an assertion stating that the derivative or variation of some functional vanishes if and only if a given equation is fulfilled. An extremum principle is one which establishes the equivalence between an equation and the fact that some functional attains an extremum value, either a maximum or a minimum.

Dual principles, also called complementary or reciprocal, form another class of variational principles which have received much attention recently, mainly due to the work of Noble and Sewell [3]. When a dual principle is available, the problem considered is formulated variationally in two different but interrelated ways. In one formulation a solution is characterized by a maximum principle and in the other by a minimum principle. The maximum and minimum values of the respective functionals are the same.

The value of dual variational principles in applications is great, because the difference between the values of both functionals for two different trial functions can be used as a measure of the accuracy of approximate solutions. In many cases the significance of dual variational principles is enhanced because the functionals involved have by themselves some relevant physical meaning, e.g. the energy. Applications of the principles have abounded and accounts of them are available [3-7].

Noble and Sewell [3] have formulated dual variational principles as generalized Lagrange and generalized Hamilton principles. Lagrange principles apply to a system of equations of the type

$$
T^{*} u=Y, \quad T(\partial Y / \partial y)=-(\partial Y / \partial u),
$$

where $T$ is a linear operator, $T^{*}$ its adjoint and $Y$ a nonlinear functional. Hamilton principles apply to the system

$$
T^{*} u=\partial X / \partial x, \quad T x=\partial X / \partial u .
$$

Here $X$ is a nonlinear functional. The extremum principles are established if some

* Received September 9, 1974; revised version received December 31, 1974.

${ }^{1}$ CIMAS (Center for Research in Applied Mathematics and Systems).

${ }^{2}$ Instituto de Ingeniería. 
functionals defined in terms of $Y$ and $X$ have properties connected with the notions of convex, concave and saddle-shaped functionals. More recently Sewell $[8,9]$ developed a framework which allows to generate this kind of principle from a single functional, thus unifying the theory.

A particular case of Sewell's results [8] implies that a variational principle formulated in terms of a single functional is a dual principle, whenever the functional is saddle. Using this result, it is possible to give a unified formulation of a large class of principles and to interrelate them [10]. For differentiable operators and functionals, such formulation can be summarized as follows.

A sufficient condition for an operator equation to admit a variational formulation is that the operator be potential, and this will be so if and only if its derivative is a symmetric bilinear functional. Variational and extremum principles are interrelated because a necessary condition for the existence of an extremum is the vanishing of the variation. Naturally, this condition is not sufficient and, therefore, the class of extremum principles is a proper subset of the class of all variational principles. A sufficient condition for a variational principle to be extremal is that the involved functional be either convex or concave $[3,10]$. In general, a functional is neither convex nor concave; however, if the linear space in which the functional is defined can be decomposed into two subspaces, one in which the functional is convex and another in which it is concave, then the functional is saddle and Sewell's results [8] can be applied; hence, the variational principle becomes a dual principle. Such a decomposition can be expected to exist under very general conditions, at least locally, because as is well known in differential geometry, this is the case for finite-dimensional spaces.

For linear operators the theory becomes especially simple: "A sufficient condition for a linear equation to admit a variational formulation is that the operator be symmetric. If the operator is either positive or negative, then the associated functional is either convex or concave, and consequently the variational principle is an extremal one. In general a symmetric linear operator is neither positive nor negative, but under very general conditions (e.g. when spectral theorems are applicable), the linear space $D$ can be decomposed into two subspaces, one $D_{+}$in which the operator is positive and another one $D_{-}$in which it is negative; thus, the associated functional is saddle and the variational principle becomes a dual variational principle of Sewell's type [8]. Accordingly, under very general conditions a dual principle can be constructed for linear equations formulated in terms of linear symmetric operators. In specific applications, the formulation of such dual principles can be complicated by the fact that the construction of the decomposition of the linear space into the subspaces $D_{+}$and $D_{-}$may be difficult. The fact that such decomposition is not unique, however, gives greater flexibility to the method."

Traditionally the theoretical foundations of variational methods have been placed on the theory of differentiation on Hilbert spaces, or more generally, on Banach spaces $[11,12]$. The formulation of variational principles can actually be carried out in linear spaces in which neither an inner product nor a norm is present. To this end it is convenient to formulate the problem in terms of functional-valued operators. Such approach has already been used in a previous work [13] and later developed systematically [10].

It is not possible to assess the importance of this result at this stage. The authors feel, however, that a systematic development of the theory is useful because:

i) Most of the work done thus far in this field [3-9, 14-18] gives the impression that an inner product or norm is required for the formulation of variational principles. In 
some applications the introduction of an inner product leads to unwarranted complications, not only in the definition of the inner product itself, but even of the function spaces considered. Indeed, many times the spaces are defined as pairs of functions, one defined in a region and the other on its boundary. This is not required when functional operators are used.

ii) The introduction of superfluous hypotheses in the development of a theory is always inconvenient, because frequently they needlessly restrict its applicability. Vainberg [12] points out, for example, that Golomb narrowed the applicability of the concept of gradient of a functional by imposing unnecessary continuity conditions. Further work by Lusternik, Sobolev, Tsilanadze and Vainberg himself was necessary before this concept was freed from such restrictions and made suitable for applications.

iii) The symmetry condition for the potentialness of an operator can be extended to linear spaces for which no inner product nor norm have to be defined-a fact not hard to prove yet not evident. This fact makes it possible to formulate a theory which is rigorous and at the same time not complicated.

After presenting a summary of this theory, in the second part of the paper we apply these advances to problems of interest in ground water hydrology.

Variational and extremum principles have been developed for steady-state problems in fluid flow through porous media. They are associated with the classical results of potential theory and elliptic differential equations. For initial-value problems the development has been less satisfactory. Only variational principles of the simplest type are available, and up to now neither extremal nor dual principles have been obtained.

The available variational principles for transient flow of ground water were formulated by Neuman and Witherspoon [19, 20] using an approach developed by Gurtin [21]. Gurtin's variational principles were originally obtained from considerations regarding the Laplace transforms of the basic differential equations, and he did not establish the connection of his results with the general theory of variational methods. Later, Sandhu and Pister [14] and Tonti [15, 16] suggested how Gurtin's approach can be set within this framework, and a general formulation of linear initial-value problems was given by Herrera and Bielak [13].

To derive variational principles for initial-value problems it is necessary to introduce the initial conditions into the governing equations. In Gurtin's method, the inverse of the time operator is applied to obtain a system of integrodifferential equations which contains the initial conditions implicitly and for which variational principles can be derived with use of convolutions. This transformation is not required if the problem is formulated in terms of functional-valued operators, the resulting variational principles [13] being simpler than those that Gurtin's method yields.

In this paper the problem of transient flow of ground water is formulated in three alternative fashions and for each reciprocal relations are established. With these, the problems are reformulated in terms of functional-valued operators which are linear and symmetric. Variational principles are then derived; they are a simplification of those due to Neuman and Witherspoon [19, 20]. Taking into account the theoretical framework discussed earlier, one would expect that it would be possible to go further and transform the variational principles into dual principles. This is indeed the case, because the linear spaces in which the problems are formulated can be decomposed into two subspaces, which are made essentially of even and odd functions with respect to the middle point of the time interval considered; in these subspaces the operator satisfies the required 
positiveness and negativeness conditions and, therefore, the stationary variational principles become dual extremum principles.

2. Mathematical preliminaries. All linear spaces to be considered will be defined on the field of real numbers $R^{1}$. The outer sum of two such spaces $D_{1}$ and $D_{2}$ will be represented by $D_{1} \oplus D_{2}$. On the other hand, if a linear space $D$ is spanned by two linearly independent subspaces $D_{1}$ and $D_{2}$, the space $D$ is isomorphic to $D_{1} \oplus D_{2}$ and we will write $D=D_{1} \oplus D_{2}$; the subspaces $D_{1}, D_{2}$ are called a decomposition of $D$. In this case given any $x \in D$ there is a unique pair of elements $\left(x_{1}, x_{2}\right)$ such that $x_{i} \in D_{i}(i=1,2)$ and $x=x_{1}+x_{2}$; this pair is called a representation of $x$. The notation $D^{n}$ will be used for the outer sum $D_{1} \oplus \cdots \oplus D_{n}$ when $D_{i}=D,(i=1, \cdots, n)$.

The value of an $n$-linear ${ }^{1}$ functional $\alpha: D^{n} \rightarrow R^{1}$ at an element $x=\left(x_{1}, \cdots, x_{n}\right) \in D^{n}$ will be represented by $\left\langle\alpha, x_{1}, \cdots, x_{n}\right\rangle^{2}$. The notation $D^{n *}$ will be used for the linear space of all the $n$-linear functionals. The alternative notation $D^{*}$ will be used for $D^{1 *}$, and $D^{\circ *}$ is defined as $R^{1}$. Notice that $D^{n *} \neq\left(D^{n}\right)^{*}$.

Functional-valued operators $P: D \rightarrow D^{n *}$ are considered in this work. Special attention will be given to the case $n=1$; i.e. to operators of the form $P: D \rightarrow D^{*}$. When $P=L$ is linear, its adjoint $L^{*}: D \rightarrow D^{*}$ is defined by $\left\langle L^{*} x, y\right\rangle=\langle L y, x\rangle$ which holds for every $x, y \in D$. Observe that the adjoint of such linear operators always exists.

For operators of the type $P: D \rightarrow D^{n *}$, the notion of continuity can be introduced without a topology in $D$. The operator $P: D \rightarrow D^{n *}$ is said to be bidimensionally continuous at $x \in D$ if for every $y, z, \xi^{(1)}, \cdots, \xi^{(n)} \in D$, the real-valued function $f(\eta, \lambda)=$ $\left\langle P(x+\eta y+\lambda z), \xi^{(1)}, \cdots, \xi^{(n)}\right\rangle$ is continuous at $\eta=\lambda=0$.

The concept of derivative of an operator will be used in the sense of additive Gateaux variation [11]. More precisely, an element $P^{\prime}(x) \in D^{(n+1) *}$ will be called the derivative of $P$ at $x \in D$, if for every $y, \xi^{(1)}, \cdots, \xi^{(n)} \in D$,

$$
g^{\prime}(0)=\left\langle P^{\prime}(x), y, \xi^{(1)}, \cdots, \xi^{(n)}\right\rangle
$$

whenever the function $g(t) \equiv\left\langle P(x+t y), \xi^{(1)}, \ldots, \xi^{(n)}\right\rangle$. Partial derivatives $P_{1}(x)$, $P,_{2}(x) \in D^{(n+1) *}$ will be considered when $D=D_{1} \oplus D_{2}$. Using the unique representation $\left(y_{1}, y_{2}\right), y_{i} \in D_{i}(i=1,2)$, of every $y \in D$, they are defined by:

$$
\left\langle P,{ }_{i}(x), y, \xi^{(1)}, \cdots, \xi^{(n)}\right\rangle=\left\langle P^{\prime}(x), y_{i}, \xi^{(1)}, \cdots, \xi^{(n)}\right\rangle, \quad(i=1,2)
$$

which holds for every $\xi^{(1)}, \cdots, \xi^{(n)} \in D$.

An operator $P: D \rightarrow D^{*}$ is said to be potential if there exists a functional $\psi: D \rightarrow$ $R^{1}=D^{\text {** }}$ such that $\psi^{\prime}(x)=P(x)$ for every $x \in D$. It is well known [12] that a sufficient condition for potentialness is that $P^{\prime}(x)$ be symmetric for every $x \in D$. This result remains valid for the class of operators $P: D \rightarrow D^{*}$ considered here if $P^{\prime}(x)$ is assumed to be bidimensionally continuous at each $x \in D$, as has been shown [10] in a manner similar to that suggested by Vainberg [12]. For a linear operator $L$, this requirement reduces to the condition that $L$ be symmetric. Such $L$ will be said to be non-negative if $\langle L x, x\rangle \geq 0$ for every $x \in D$ and positive if in addition $\langle L x, x\rangle=0$ only when $x=0$. Non-positive and negative operators are defined similarly.

${ }^{1}$ Russian authors usually include continuity in the definition of linearity [12]. On the other hand, for most American authors this concept includes only additivity and homogeneity. In this paper we follow American usage.

2 This notation does not imply the existence of an inner product. As a matter of fact, no inner product or norm will be used in this paper. 
The general problem to be considered consists in finding solutions to an equation

$$
P(x)=f
$$

where $P$ is a functional-valued operator $P: D \rightarrow D^{*}$ and $f \in D^{*}$. The solutions $x$ will be restricted to be in a subset $\hat{E} \subset D$. The elements belonging to $\hat{E}$ satisfy some additional boundary or initial conditions and are called admissible states. It will be assumed that they constitute an affine subspace; i.e., there is a subspace $E \subset D$ and an element $w \in D$ such that $\hat{E}=w+E$. Clearly, $E=\hat{E}$ when $w \in E$.

Given a functional $X: D \rightarrow R^{1}$, for each $x \in \hat{E}$ we define the variation of $X$ at $x$ as a linear functional $\delta X(x) \in E^{*}$, such that

$$
\langle\delta X(x), y\rangle=\left\langle X^{\prime}(x), y\right\rangle
$$

for every $y \in E$. When a decomposition $E_{1}, E_{2}$ of $E$ is available, at every $x \in \hat{E}$ the partial variations $\delta_{i} X(x) \in E_{i}{ }^{*}(i=1,2)$ are defined in a manner similar to partial derivatives.

Following Noble and Sewell [3], given any two elements $x_{-}, x_{+} \in \hat{E}$ define

$$
\begin{aligned}
\Delta X & =X\left(x_{+}\right)-X\left(x_{-}\right), \\
\Delta x & =x_{+}-x_{-} .
\end{aligned}
$$

Observe that $\Delta x \in E$. Thus, if a decomposition $E_{1}, E_{2}$ of $E$ is available, let $\Delta_{i} x \in E_{i}$ $(i=1,2)$ be the unique representation of $\Delta x$ in terms of an element of $E_{1}$ plus an element of $E_{2}$.

When the functional $X$ is differentiable, it is said to be convex on $\widehat{E}$, if

$$
\Delta X-\left\langle\delta X\left(x_{-}\right), \Delta x\right\rangle \geq 0
$$

or equivalently

$$
\Delta X-\left\langle\delta X\left(x_{+}\right), \Delta x\right\rangle \leq 0
$$

for every $x_{+}, x_{-} \in \hat{E}$. It is strictly convex if the strict inequality holds whenever $x_{+} \neq x_{-}$. In addition, $X$ is concave or strictly concave if $-X$ is convex or strictly convex respectively.

Furthermore, $X$ is saddle on $\widehat{E}$, convex on $E_{1}$ and concave on $E_{2}$ if

$$
\Delta X-\left\langle\delta_{1} X\left(x_{-}\right), \Delta_{1} x\right\rangle-\left\langle\delta_{2} X\left(x_{+}\right), \Delta_{2} x\right\rangle \geq 0
$$

for every $x_{+}, x_{-} \in \hat{E}$. It is strictly saddle if the inequality is strict whenever $x_{+} \neq x_{-}$.

3. Formulation of variational principles. By a variational principle is understood an assertion stating that the variation $\delta X(x)$ of a functional $X$ vanishes at a point $x \in E$ if and only if $x$ is a solution of (3). When $\hat{E}=D$, a sufficient condition for the construction of variational principles is that the operator $P$ be potential, because if $\psi: D \rightarrow R^{1}$ is a potential of $P$, then the functional $X(x)=\psi(x)-\langle f, x\rangle$ will have the required property.

Variational principles which hold when the set of admissible states is $D$ itself but which remain valid when the set of admissible states is restricted to be $\hat{E} \subset D$ are known in many instances. The validity of such procedure frequently depends on the next obvious fact. 
Lemma 1. Assume

i) $X^{\prime} \equiv P-f$ where $f \in D^{*}$;

ii) For every $x \in \hat{E},\langle P(x)-f, y\rangle=0$ for every $y \in E$, if and only if (3) is satisfied. Then for every $x \in \hat{E}, X^{\prime}(x)=0$ if and only if $\delta X(x)=0$.

An element $x_{0} \in D$ is said to be a maximum of a functional $X: D \rightarrow R^{1}$ if

$$
X\left(x_{0}\right) \geq X(x)
$$

for every $x \in D$. The maximum is said to be strict if the inequality holds whenever $x \neq x_{0}$. Minimum and strict minimum are defined similarly.

Obviously, if $X$ possesses a derivative at $x_{0}$, then $X^{\prime}\left(x_{0}\right)=0$ whenever $x_{0}$ is a maximum or a minimum of $X$. Conversely [3], if $X$ is convex or concave, then $x_{0}$ is a maximum or a minimum respectively of $X$ whenever $X^{\prime}\left(x_{0}\right)=0$. The maximum or minimum will be strict if the convexity or concavity are strict.

In general, a functional $X$ is neither convex nor concave, but under very general conditions can be expected to be saddle-shaped-bilinear functionals illustrate this fact, as will be seen. For this reason, the extension by Sewell [8] of the foregoing results to saddle-shaped functionals enlarges considerably the applicability of dual extremum principles. Our version of a special case of Sewell's results is contained in the following theorem and corollary.

First recall that

$$
\delta X(x) \equiv \delta_{1} X(x)+\delta_{2} X(x)=0
$$

at $x \in \hat{E}$ if and only if

$$
\delta_{1} X(x)=0
$$

and simultaneously

$$
\delta_{2} X(x)=0 .
$$

Theorem 2. Assume a decomposition $E_{1}, E_{2}$ of $E$ is available and $X: D \rightarrow R^{1}$ is a saddle functional on $\hat{E}$, convex in $E_{1}$ and concave in $E_{2}$. Then

i) For any $x_{a}, x_{b} \in E$ that satisfy (11a) and (11b) respectively, we have

$$
X\left(x_{a}\right) \leq X\left(x_{b}\right)
$$

ii) if $x \in E$ is a solution of (10), then:

$\alpha)$ The maximum value of $X$ among all admissible states that satisfy (11a) is attained at $x$;

$\beta$ ) The minimum value of $X$ among all admissible states that satisfy (11b) is attained at $x$;

$\gamma)$ The respective maximum and minimum values coincide.

Proof. This theorem was established by Sewell [8], but because of its simplicity the proof is presented herein for the sake of completeness. To prove part (i), observe that relation (8) is satisfied by any given pair of admissible states $x_{+}$and $x_{-}$. Thus, if we set $x_{+}=x_{b}$ and $x_{-}=x_{a}$, relation (12) follows. Now, if $x$ is a solution of (10), then $x$ satisfies Eqs. (11); therefore $(\alpha)$ and $(\beta)$ follow from (12). Finally, $\gamma$ ) is a direct consequence of $(\alpha)$ and $(\beta)$.

An attractive feature of many dual principles is that any member $x_{a}$ satisfying (11a) provides an upper bound and any member $x_{b}$ satisfying (11b) supplies a lower bound 
of the common value of the functional $X$ at the solution $x$. The results of Theorem 2, however, do not allow to infer from the difference $X\left(x_{a}\right)-X\left(x_{b}\right)$ an estimate of the closeness between an approximate solution and an exact solution. Theorem 2 states that the minimum value of the functional $X$ on solutions of (11a) is equal to its maximum value on solutions of $(11 \mathrm{~b})$, when a solution $x$ of (10) exists. Though in this case both maximum and minimum are attained at $x$, there may be elements $x^{\prime}$ satisfying either (11a) or (11b) on which $X$ achieves the same value and which are not solutions of (10). This difficulty does not arise if $X$ is strictly saddle, as the following corollary shows.

Corollary 3. If in Theorem $2 X$ is strictly saddle on $\hat{E}$,

i) There is at most one solution $x \in \hat{E}$ of Eq. (10);

ii) The equation

$$
X\left(x_{a}\right)=X\left(x_{b}\right)
$$

holds if and only if $x_{a}=x_{b}$ is the solution of (10);

iii) If the solution of (10) belongs to $\hat{E}$, the maximum value of $X$ among all admissible states that satisfy (11a) is attained exclusively at the solution. The minimum value of the functional among all admissible states that fulfill (11b) also is attained only at the solution.

Proof. In this case the proof of part (i) of Theorem 2 yields (ii). The result (i) is obviously implied by (ii). Finally, to prove (iii), let $x$ be the solution of (10) and let $X\left(x_{a}\right)$ be the maximum value of $X$ among all admissible states that satisfy (11a); then

$$
X\left(x_{a}\right)=X(x)
$$

and consequently $x_{a}=x$. The proof of the second part of (iii) is similar.

This corollary, and in particular parts (ii) and (iii), are of special relevance for the construction of approximate solutions because they show that the elements that yield the maximum and the minimum are unique and equal to each other and correspond to the solution of the problem. This condition is required to assure that the difference $X\left(x_{\imath}\right)-X\left(x_{a}\right)$ can be used as an estimate of the error of an approximate solution. Thus, the practical usefulness of the results is greater for problems associated with functionals that are strictly saddle.

4. Dual variational principles for linear symmetric operators. An advantage of introducing the class of variational principles formulated in Theorem 2 and Corollary 3 is that this class has a clear connection with other kinds of principles, thus permitting a unified formulation of the theory. For the linear case such procedure yields a general class of dual variational principles applicable to symmetric operators under very general conditions [10].

Let $L: D \rightarrow D^{*}$ be a linear and symmetric functional-valued operator. The condition of symmetry on $L$ implies that the operator $L$ is potential, and therefore the equation

$$
L x=f,
$$

with $f \in D^{*}$, admits a variational formulation. Indeed, Eq. (15) is equivalent to $X^{\prime}(x)=0$, where

$$
X(x)=\frac{1}{2}\langle L x, x\rangle-\langle f, x\rangle
$$


On the class of admissible states $\hat{E}$, Eq. (15) will be equivalent to $\delta X(x)=0$ as long as the hypotheses of Lemma 1 are satisfied. This variational principle is extremal if the class of admissible states $\hat{E}$ is such that $L$ is non-negative or non-positive on $\hat{E}$. When this is not the case, the fact that a linear space $E$ can, under very general conditions, be decomposed into two subspaces $E_{1}, E_{2}$ such that $L$ is non-negative on $E_{1}$ and non-positive on $E_{2}$ can be used to construct dual variational principles. For example, if $E$ possesses a basis of eigenvectors, $E_{1}$ and $E_{2}$ can be taken as the subspaces generated by the eigenvectors corresponding to positive and non-positive eigenvalues, respectively. With this decomposition the functional $X$ is saddle on $\hat{E}$, convex on $E_{1}$ and concave on $E_{2}$; Theorem 2 is therefore applicable. If $L$ is positive on $E_{1}$ and negative on $E_{2}$, $X$ is strictly saddle and Corollary 3 also can be applied.

Observe there are other ways in which the decomposition of $E$ can be carried out This is a useful fact because it adds flexibility to the procedure in specific applications.

5. On the treatment of boundary conditions. The formulation of variational principles presented here is applicable to an equation of the form (3) which is defined in terms of functional-valued operators. To apply it to systems of partial differential equations, it is therefore necessary to express them first in this form. Boundary conditions that have been treated by other authors $[3,17,18]$ can be included in the present framework and the general ideas of the procedure are explained herein. The variational principles developed in the following sections, as well as some obtained previously [13], illustrate the way in which it can be used.

Let $D$ be a linear space of functions defined on a compact connected subset $R$ of $R^{n}$ with smooth boundary $\partial R$. Let $\hat{L}: D \rightarrow D^{*}$ be a linear differential operator. A formal adjoint $\hat{L}$ is an operator $\hat{L}^{*}: D \rightarrow D^{*}$ such that for any $x, y \in D$ :

$$
\langle\hat{L} x, y\rangle=\left\langle\hat{L}^{*} y, x\right\rangle+\phi(x, y)
$$

where $\phi: D^{2} \rightarrow R^{1}$ is a functional whose support is contained in $\partial E$. In applications, an equation of the form (17) is usually given by a reciprocal theorem (see $[3,13])$ and in that case $\langle\hat{L} x, y\rangle$ is given in terms of a differential operator applied to $x$, integrated after multiplication by $y .\left\langle\hat{L}^{*} y, x\right\rangle$ is given similarly. Clearly, $\phi$ is a bilinear functional because $\langle\hat{L} x, y\rangle$ and $\left\langle\hat{L}^{*} y, x\right\rangle$, are bilinear functionals (compare with Komkov [17] and Arthurs [18]). It is therefore possible to define operators $\hat{B}: D \rightarrow D^{*}$ and $\hat{B}^{*}: D \rightarrow D^{*}$ with support in $\partial E$ such that for every $x, y \in D$ :

$$
\langle\hat{L} x, y\rangle+\langle\hat{B} x, y\rangle=\left\langle\hat{L}^{*} y, x\right\rangle+\left\langle\hat{B}^{*} y, x\right\rangle .
$$

This can be done in many ways, because all that is required is that

$$
\left\langle\hat{B}^{*} y, x\right\rangle-\langle\hat{B} x, y\rangle=\phi(x, y)
$$

for every $x, y \in D$. However, if the operator $L$ in (15) is to be defined by $L=\hat{L}+\hat{B}$, there must be a one-to-one correspondence between the range of the operator $\hat{L}+$ $\hat{B}: D \rightarrow D^{*}$ and the possible data of the problem. A necessary and sufficient condition is that $(\hat{L}+\hat{B}) x$ vanishes if and only if $x$ satisfies null data. In particular, $\hat{B} x$ vanishes if and only if $x$ satisfies zero boundary data. If this is achieved, $f \in D^{*}$ defined by $f=\hat{f}+\hat{f}_{B}$ is known when the data of the problem are specified and the problem can be formulated as in (15), where $L=\hat{L}+\hat{B}, \hat{f}=\hat{L} x$ and $\hat{f}_{B}=\hat{B} x$. 
It must be observed that there is not a unique manner of formulating the problem in terms of functional-valued operators and consequently, given one variational principle, other principles can be associated with the same problem. Indeed, let $P: D \rightarrow D^{*}$ be self-adjoint such that $P x=0$ if and only if $x$ satisfies zero boundary conditions. Then, if $L$ is self-adjoint, $L+a P$ is self-adjoint for every $a \in R^{1}$ and it can be seen that

$$
(L+a P) x=f+a g
$$

is equivalent to (15), for some $a \neq 0$. Here $g \in D^{*}$ is given by $g=P x$.

6. Alternative formulations of the flow problem. The boundary initial-value problem to be studied is the transient flow of water in a confined flow region, i.e. a completely saturated elastic porous medium that has well-defined geometric boundaries. It is assumed that an open region $R$ with boundary $A$ is occupied by a porous and permeable medium completely filled with a slightly compressible liquid such as water or oil. The medium through which the flow occurs has a specific storage $S_{s}(\mathbf{x})$ and a symmetric permeability tensor $K_{i j}(\mathbf{x})$, properties which are dependent upon the position vector $\mathbf{x} . S_{8}(\mathbf{x})$ is positive and continuous on $\bar{R}$ and $K_{i i}(\mathbf{x})$ is assumed to be positive definite and continuously differentiable on $\bar{R}$. The problem consists in finding the dynamic state of the liquid at any instant when an initial state and boundary data are known. The boundary data are given in two complementary parts $A_{1}$ and $A_{2}$ of $A . A_{1}$ is the portion of the boundary on which the head is prescribed and $A_{2}$ is the remaining portion of the boundary, on which flux is prescribed.

We do not discuss the existence of solutions, assuming, therefore, that a solution exists, i.e. that the data are in the range of the operator.

Three alternative descriptions of the motion will be considered depending on the variables used to characterize the problem:

i) Equations in terms of head and velocity. Let $h$ be the hydraulic head and $v_{i}$ denote the cartesian components of the Darcy velocity vector $\mathbf{v}$. The physical laws governing the motion of the fluid are the equation of continuity

$$
S_{s}(\partial h / \partial t)+\left(\partial v_{i} / \partial x_{i}\right)=q \text { on } R
$$

and Darcy's law

$$
v_{i}=-K_{i i} \frac{\partial h}{\partial x_{i}} \text { on } R .
$$

These equations are supplemented by the initial condition

$$
h(\mathbf{x}, 0)=h_{0}(\mathbf{x}) \quad \text { on } R
$$

and the boundary conditions

$$
\begin{aligned}
h(\mathbf{x}, t) & =H(\mathbf{x}, t) \quad \text { on } \quad A_{1}, \\
v_{i}(\mathbf{x}, t) n_{i}(\mathbf{x}) & =W(\mathbf{x}, t) \text { on } A_{2} .
\end{aligned}
$$

In Eqs. (21), $q(\mathbf{x}, t)$ denotes the external sources, the function $h_{0}$ is the prescribed initial head, while $H$ and $W$ are given surface head and surface flux. $n_{i}$ are the cartesian components of the outward unit normal vector $\mathbf{n}$. Here and in what follows the range of Latin subscripts will be $\{1,2,3\}$ and sum over repeated indices is understood. 
Usually the problem is posed either on an infinite time interval, i.e., for every $t \geq 0$, or, alternatively, on a finite time interval. Here the problem will be formulated on the interval $\left[0, t_{1}\right]$. Thus, Eqs. (21a), (21b), (21d) and (21e) will need to be satisfied for every $t \in\left[0, t_{1}\right]$.

In this formulation the set $D$ of states will consist of all possible systems of functions $\{h(\mathbf{x}, t), \mathbf{v}(\mathbf{x}, t)\}$ defined on $\bar{R} \times\left[0, t_{1}\right]$ such that $h$ together with its first spatial and time derivatives are continuous everywhere in $R$, while $v_{i}$ are continuous in space and time and have continuous first spatial derivatives. This space will be denoted by $D_{c}$. Neuman and Witherspoon [19,20] have discussed some of the advantages gained by formulating the flow problem as in Eqs. (21), which permit evaluating head and Darcy velocity simultaneously.

ii) Equations in terms of head. In dealing with flow in porous media it has been customary in hydrology to characterize the problem only in terms of head. To arrive at such formulation it is necessary to make use of Eq. (21b) to eliminate $v_{i}$ from the remaining equations in the system (21). This process leads to

$$
\begin{gathered}
S_{s} \frac{\partial h}{\partial t}=\frac{\partial}{\partial x_{i}}\left(K_{i i} \frac{\partial h}{\partial x_{i}}\right)=q \text { on } R \times\left[0, t_{1}\right], \\
h(\mathbf{x}, 0)=h_{0}(\mathbf{x}) \quad \text { on } R, \\
h(\mathbf{x}, t)=H(\mathbf{x}, t) \quad \text { on } A_{1} \times\left[0, t_{1}\right] \\
K_{i j} \frac{\partial h}{\partial x_{i}} n_{i}=-W(\mathbf{x}, t) \text { on } A_{2} \times\left[0, t_{1}\right] .
\end{gathered}
$$

The set $D$ of states for this formulation will be made of the continuous functions $h(\mathbf{x}, t)$ defined on $\bar{R} \times\left[0, t_{1}\right]$, such that their second space derivatives and first time derivatives are continuous everywhere on $R \times\left[0, t_{1}\right]$, and will be referred to as $D_{h}$.

iii) Equations in terms of velocity. The hydraulic head may be eliminated from Eqs. (21) to obtain a characterization of the flow problem in terms of velocity [19]. The resulting system is:

$$
K_{i j}{ }^{-1} \frac{\partial v_{i}}{\partial t}-\frac{\partial}{\partial x_{i}}\left(\frac{1}{S_{s}} \frac{\partial v_{i}}{\partial x_{i}}\right)=p_{i}(\mathbf{x}, t) \quad \text { on } \quad R \times\left[0, t_{1}\right]
$$

where $K_{i j}{ }^{-1}$ are the elements of the inverse of $K_{i i}$, i.e., $K_{i j}{ }^{-1} K_{i k}=\delta_{i k}$. The corresponding boundary and initial conditions are:

$$
\begin{array}{cc}
v_{i}(\mathbf{x}, 0)=N_{i}(\mathbf{x}), & \mathbf{x} \in R, \\
\frac{1}{S_{s}} \frac{\partial v_{i}}{\partial x_{i}}=M(\mathbf{x}, t) \quad \text { on } \quad & A_{1} \times\left[0, t_{1}\right], \\
v_{i} n_{i}=W(\mathbf{x}, t) \quad \text { on } & A_{2} \times\left[0, t_{1}\right] .
\end{array}
$$

The functions $p_{i}(\mathbf{x}, t), N_{i}(\mathbf{x})$ and $M(\mathbf{x}, t)$ appearing in these equations need to be prescribed. If the problem is formulated originally in terms of head and velocity, these functions are given by

$$
p_{i}(\mathbf{x}, t)=-\frac{\partial}{\partial x_{i}}\left[\frac{1}{S_{s}(\mathbf{x})} q(\mathbf{x}, t)\right] \text { on } R \times\left[0, t_{1}\right]
$$




$$
\begin{aligned}
N_{i}(\mathbf{x}) & =-K_{i j}(\mathbf{x}) \frac{\partial h_{o}(\mathbf{x})}{\partial x_{i}}, \quad \mathbf{x} \in R \\
M(\mathbf{x}, t) & =-\frac{\partial H(\mathbf{x}, t)}{\partial t}+q(\mathbf{x}, t) \quad \text { on } A_{1} \times\left[0, t_{1}\right]
\end{aligned}
$$

The set $D$ of states will be called $D_{v}$ and will consist of the continuous functions $v_{i}(\mathbf{x}, t)$ defined on $\bar{R} \times\left[0, t_{1}\right]$ such that their second space derivatives and first time derivatives are continuous everywhere on $R$. The hydraulic head does not enter into this formulation but Eq. (21a) can be used to define it.

7. Variational principles for the flow problem. To derive the variational principles associated with the flow problems under study by use of the results presented in the previous sections, it is first necessary to reformulate these problems in terms of functional-valued operators. The aim here is to cast each of the three flow problems in the form of Eq. (15), with a symmetric operator $L$. This accomplished, it is then a simple matter to establish the desired variational principles.

In the remainder of this paper the letter $x$ will be reserved to denote the space coordinates. Specific notations for the elements of the linear spaces of states will be given in each section.

i) Formulation in terms of head and velocity. A functional-valued operator associated with this formulation can be obtained from the following reciprocity relation.

Theorem 3. Let $U=\{h, \mathbf{v}\}$ and $V=\{\bar{h}, \overline{\mathbf{v}}\}$ be two elements of the set $D_{c}$ of states defined in Section 6 i). Then

$$
\begin{array}{r}
\int_{R}\left[\bar{h} * S_{s} \frac{\partial h}{\partial t}+\bar{h} * \frac{\partial v_{i}}{\partial x_{i}}-\bar{v}_{i} * \frac{\partial h}{\partial x_{i}}-\bar{v}_{i} * K_{i j}{ }^{-1} v_{i}+S_{s} h(\mathbf{x}, 0) \bar{h}\left(\mathbf{x}, t_{1}\right)\right] d \mathbf{x} \\
\quad+\int_{A_{i}} \bar{v}_{i} n_{s} * h d \mathbf{x}-\int_{A_{2}} \bar{h} * v_{i} n_{i} d \mathbf{x} \\
=\int_{R}\left[h * S_{s} \frac{\partial \bar{h}}{\partial t}+h * \frac{\partial \bar{v}_{i}}{\partial x_{i}}-v_{i} * \frac{\partial \bar{h}}{\partial x_{i}}-v_{i} * K_{i j}{ }^{-1} \bar{v}_{i}+S_{s} \bar{h}(\mathbf{x}, 0) h\left(\mathbf{x}, t_{1}\right)\right] d \mathbf{x} \\
+\int_{A_{1}} v_{i} n_{i} * h d \mathbf{x}-\int_{A_{2}} h * \bar{v}_{i} n_{i} d x
\end{array}
$$

A notation motivated by the convolution notation has been adopted in this equation, i.e., for every pair of functions $k, g$ :

$$
(k * g)(\mathbf{x})=\int_{0}^{t_{1}} k(\mathbf{x}, t) g\left(\mathbf{x}, t_{1}-t\right) d t .
$$

Proof. Integration by parts with respect to $t$ and use of the divergence theorem on the left-hand member of (25) yields directly the right-hand member.

Given $U=\{h, \nabla\} \in D_{c}$, the left-hand side of Eq. (25) defines a linear functional of $V=\{\bar{h}, \overline{\mathbf{v}}\} \in D_{c}$. The functional so defined is identically zero if and only if $U$ satisfies null data. Therefore, according to the remarks of Sec. 5, the functional-valued operators $\hat{L}, \hat{B}: D_{c} \rightarrow D_{c}^{*}$ can be defined by

$$
\begin{aligned}
& \langle\hat{L} U, V\rangle=\int_{R}\left[\bar{h} * S_{\mathrm{s}} \frac{\partial h}{\partial t}+\bar{h} * \frac{\partial v_{i}}{\partial x_{i}}-\bar{v}_{i} * \frac{\partial h}{\partial x_{i}}-\bar{v}_{i} * K_{i i}{ }^{-1} v_{i}\right] d \mathbf{x} \\
& \langle\hat{B} U, V\rangle=\int_{R} S_{\mathrm{s}} h(\mathbf{x}, 0) \bar{h}\left(\mathbf{x}, t_{1}\right) d \mathbf{x}+\int_{A_{1}} \bar{v}_{i} n_{i} * h d \mathbf{x}-\int_{A_{2}} \bar{h} * v_{i} n_{i} d x
\end{aligned}
$$


which hold for every $U, V \in D_{c}$. Applying these equations to a solution of the system (21) leads to defining $\hat{f}$ and $\hat{f}_{B}$ by

$$
\langle\hat{f}, V\rangle=\int_{R} h * q d \mathbf{x}
$$

and

$$
\left\langle\hat{f}_{B}, V\right\rangle=\int_{R} S_{s} h_{0}(\mathbf{x}) h\left(\mathbf{x}, t_{1}\right) d \mathbf{x}+\int_{A_{1}} \bar{v}_{i} n_{i} * H d \mathbf{x}-\int_{A_{z}} \bar{h} * W d \mathbf{x}
$$

which hold for every $V \in D_{c}$. Defining now $L$ as $\hat{L}+\hat{B}$ and $f$ as $\hat{f}+\hat{f}_{B}$, and using arguments similar to those presented by Herrera and Bielak [13] which are standard in calculus of variations, it can be shown that Eq. (15) is fulfilled if and only if Eqs. (21) are satisfied. Since $L$ clearly is symmetric, we have

Theorem 4. Let the set of admissible states be $\hat{E}=E=D_{c}$. For every element $\{h, \mathbf{v}\} \in D_{c}$ define the functional

$$
\begin{aligned}
& X(h, \mathbf{v})=\frac{1}{2} \int_{R}\left\{S_{\mathrm{s}} h * \frac{\partial h}{\partial t}-2 v_{i} * \frac{\partial h}{\partial x_{i}}-v_{i} * K_{i i}{ }^{-1} v_{i}+S_{\mathrm{s}} h\left(\mathbf{x}, t_{1}\right)\right. \\
& \left.\cdot\left[h(\mathbf{x}, 0)-2 h_{0}(\mathbf{x})\right]-2 h * q\right\} d \mathbf{x}+\int_{A_{1}}(h-H) * v_{i} n_{i} d \mathbf{x}+\int_{A} h * W d \mathbf{x} .
\end{aligned}
$$

Then

$$
\delta X(h, \mathbf{v})=0
$$

if and only if $\{h, \mathbf{v}\}$ is a solution of the problem specified by Eqs. (21).

Proof. Substitution of $L$ and $f$ for this problem into (16) gives (30). On the other hand, $X^{\prime}=\delta X$ because $\hat{E}=D_{c}$.

The functional defined by Eq. (30) is simpler, and slightly more general, than that given by Neuman and Witherspoon [19]. The latter is obtained when the former is convoluted with the constant function -1 , if $q$ is set equal to zero [13].

To obtain the dual variational principle associated with the present formulation of the problem, define $E_{1}$ as the subset of $E$ whose elements $\{h, \mathbf{v}\}$ are such that $h$ is even while $\mathbf{v}$ is odd about the midpoint in the interval $\left[0, t_{1}\right]$, and define $E_{2}$ as the subset of $E$ whose elements $\{h, \mathbf{v}\}$ are such that $h$ is odd while $\mathbf{v}$ is even in the same interval. With these definitions $E_{1}, E_{2}$ is a decomposition of $E$. The dual variational principle follows from Theorem 2 because $L$ is non-negative in $E_{1}$ and non-positive in $E_{2}$, as can be verified by direct substitution in the definition of $L$.

Theorem 5. Let the set $\hat{E}$ of admissible states and the functional $X$ be defined as in Theorem 4. Then

i) An admissible state $\{h, \mathbf{v}\}$ is a solution of the system (21) if and only if

$$
\begin{aligned}
& {\left[S_{s} \frac{\partial h}{\partial t}+\frac{\partial v_{i}}{\partial x_{i}}-q\right]^{e}=0 \text { on } R \times\left[0, t_{1}\right] \text {, }} \\
& {\left[v_{i}+K_{i i} \frac{\partial h}{\partial x_{i}}\right]^{\circ}=0 \text { on } R \times\left[0, t_{1}\right] \text {, }} \\
& h(\mathbf{x}, 0)=h_{o}(\mathbf{x}), \quad \mathbf{x} \in R, \\
& {[h-H]^{\circ}=0 \text { on } A_{1} \times\left[0, t_{1}\right],} \\
& {\left[v_{i} n_{i}-W\right]^{e}=0 \text { on } A_{2} \times\left[0, t_{1}\right]}
\end{aligned}
$$


and simultaneously

$$
\begin{aligned}
& {\left[S_{s} \frac{\partial h}{\partial t}+\frac{\partial v_{i}}{\partial x_{i}}-q\right]^{0}=0 \quad \text { on } R \times\left[0, t_{1}\right] \text {, }} \\
& {\left[v_{i}+K_{i j} \frac{\partial h}{\partial x_{i}}\right]^{e}=0 \text { on } R \times\left[0, t_{1}\right] \text {, }} \\
& h(\mathbf{x}, 0)=h_{o}(\mathbf{x}), \quad \mathbf{x} \in R, \\
& {[h-H]^{e}=0 \text { on } A_{1} \times\left[0, t_{1}\right] \text {, }} \\
& {\left[v_{i} n_{i}-W\right]^{\circ}=0 \text { on } A_{2} \times\left[0, t_{1}\right] .}
\end{aligned}
$$

The superscripts $e$ and $o$ denote, respectively, the even and odd components about the midpoint in the interval $\left[0, t_{1}\right]$ of the terms enclosed in brackets.

ii) For any pair of admissible states $\left\{h_{a}, \mathbf{v}_{a}\right\},\left\{h_{b}, \mathbf{v}_{b}\right\}$ that satisfy (32a) and (32b), respectively, we have

$$
X\left(h_{a}, \mathbf{v}_{a}\right) \leq X\left(h_{b}, \mathbf{v}_{b}\right)
$$

iii) Let $\{h, \mathbf{v}\}$ be an admissible state that satisfies (21). Then

$\alpha$ ) The maximum value of $X$ among all admissible states that satisfy (32a) is attained at $\{h, \mathbf{v}\}$;

$\beta$ ) The minimum value of $X$ among all admissible states that satisfy (32b) is attained at $\{h, \mathbf{v}\}$;

r) The respective maximum and minimum values coincide.

Proof. The theorem follows from Theorem 2 in view of the remarks of Sec. 4; it is only necessary to show that (32a, b) are equivalent to $(11 \mathrm{a}, \mathrm{b})$, respectively.

To this end observe that for every $U, V \in D_{c}$ we have

$$
\begin{aligned}
& \langle\delta X(U), V\rangle=\langle L U-f, V\rangle=\int_{R}\left\{h *\left[S_{s} \frac{\partial h}{\partial t}+\frac{\partial v_{i}}{\partial x_{i}}-q\right]-\bar{v}_{i} *\left[\frac{\partial h}{\partial x_{i}}+K_{i j}{ }^{-1} v_{j}\right]\right. \\
& \left.\quad+S_{s} h\left(x, t_{1}\right)\left[h(\mathbf{x}, 0)-h_{0}(x)\right]\right\} d \mathbf{x}+\int_{A_{2}} \bar{v}_{i} n_{i} *(h-H) d \mathbf{x} \\
& \quad-\int_{A_{2}} h *\left[v_{i} n_{i}-W\right] d x .
\end{aligned}
$$

Eq. (11a) states that the partial variation $\delta_{1} X$ vanishes. This condition is equivalent to requiring that $\langle\delta X(U), V\rangle$ vanish whenever $V \in E_{1}$. In view of (34), this is equivalent to (32a). Eqs. (32b) can be established similarly.

Corresponding results for the other two descriptions of the problem can be derived in a similar manner and are given in the following paragraphs. Therefore, the proofs are only sketched.

ii) Formulation in terms of head.

Theorem 6 (Reciprocity relation). Let $h$ and $\bar{h}$ be two elements of the set $D_{h}$ of states defined in Sec. 6 (ii). Then:

$$
\begin{aligned}
\int_{R}\left[h * S_{s} \frac{\partial h}{\partial t}-\bar{h}\right. & * \frac{\partial}{\partial x_{i}}\left(K_{i i} \frac{\partial h}{\partial x_{i}}+S_{s} h(\mathbf{x}, 0) \bar{h}\left(\mathbf{x}, t_{1}\right)\right] d x \\
& -\int_{A_{i}} h * K_{i i} \frac{\partial h}{\partial x_{i}} n_{i} d \mathbf{x}+\int_{A_{2}} h * K_{i j} \frac{\partial h}{\partial x_{i}} n_{i} d \mathbf{x}
\end{aligned}
$$




$$
\begin{aligned}
= & \int\left[h * S_{s} \frac{\partial \bar{h}}{\partial t}-h * \frac{\partial}{\partial x_{i}}\left(K_{i ;} \frac{\partial \bar{h}}{\partial x_{i}}\right)+S_{\mathrm{s}} h(\mathbf{x}, 0) h\left(\mathbf{x}, t_{1}\right)\right] d \mathbf{x} \\
& -\int_{A_{1}} \bar{h} * K_{i i} \frac{\partial h}{\partial x_{i}} n_{i} d \mathbf{x}+\int_{A_{2}} h * K_{i j} \frac{\partial \bar{h}}{\partial x_{i}} n_{i} d \mathbf{x} .
\end{aligned}
$$

The functional-valued operators $L, B: D \rightarrow D^{*}$ corresponding to this reciprocity relation are defined by

$$
\begin{aligned}
& \langle\hat{L} h, h\rangle=\int_{R}\left[\bar{h} * S_{s} \frac{\partial h}{\partial t}-h * \frac{\partial}{\partial x_{i}}\left(K_{i j} \frac{\partial h}{\partial x_{i}}\right)\right] d x \\
& \langle\hat{B} h, h\rangle=\int_{R} S_{s} h(\mathbf{x}, 0) h\left(\mathbf{x}, t_{1}\right) d \mathbf{x}-\int_{A_{1}} h * K_{i j} \frac{\partial h}{\partial x_{i}} n_{i} d \mathbf{x}+\int_{A_{2}} h * K_{i i} \frac{\partial h}{\partial x_{i}} n_{i} d \mathbf{x}
\end{aligned}
$$

which hold for every $h, \bar{h} \in D$.

The functionals $\hat{f}$ and $\hat{f}_{B}$ can be defined by applying Eqs. (36) and (37) to a solution $h$ of the system (22):

$$
\begin{gathered}
\langle\hat{\mathrm{f}}, \bar{h}\rangle=\int_{R} h * q d \mathbf{x} \\
\left\langle\hat{f}_{B}, \bar{h}\right\rangle=\int_{R} S_{\mathrm{s}} h_{0}(x) \bar{h}(\mathbf{x}, t)-\int_{A_{2}} h * W d \mathbf{x}-\int_{A_{1}} H * K_{i_{i}} \frac{\partial \bar{h}}{\partial x_{i}} n_{i} d \mathbf{x}
\end{gathered}
$$

for every $\bar{h} \in D$.

If we define then $L=\hat{L}+\hat{B}$ and $f=\hat{f}+\hat{f}_{B}$, arguments similar to those used in [13] can again be used to prove that an element $h \in D_{h}$ will be a solution of the system (22) if and only if Eq. (15) is satisfied.

Taking into account that $L: D_{h} \rightarrow D_{h}{ }^{*}$ so defined clearly is symmetric, a stationary variational principle follows when the functional $X$ is defined as in (16).

THEorem 7. Let the set $\hat{E}$ of admissible states coincide with the whole set $D_{h}$ of states. For every state $h$, define the functional $X$ by

$$
\begin{gathered}
X(h)=\frac{1}{2} \int_{R}\left\{S_{s} h * \frac{\partial h}{\partial t}+K_{i ;} \frac{\partial h}{\partial x_{i}} * \frac{\partial h}{\partial x_{i}}+S_{s} h\left(\mathbf{x}, t_{1}\right)\left[h(\mathbf{x}, 0)-2 h_{0}(\mathbf{x})\right]-2 h * q\right\} d \mathbf{x} \\
-\int_{A_{2}}(h-H) * K_{i j} \frac{\partial h}{\partial x_{i}} n_{i} d \mathbf{x}+\int_{A_{2}} h * W d \mathbf{x} .
\end{gathered}
$$

Then

$$
\delta X(h)=0
$$

if and only if $h$ is a solution of the problem specified by Eqs. (22).

Corollary 8. The variational principle of Theorem 7 remains valid if the set $\hat{E}$ of admissible states is restricted to satisfy the boundary condition (22c) and the functional $X$ is given by

$$
\begin{aligned}
X(h)=\frac{1}{2} \int_{R}\left\{S_{s} h\right. & * \frac{\partial h}{\partial t}+K_{i i} \frac{\partial h}{\partial x_{i}} * \frac{\partial h}{\partial x_{i}} \\
& \left.+S_{s} h\left(\mathbf{x}, t_{1}\right)\left[h(\mathbf{x}, 0)-2 h_{0}(\mathbf{x})\right]-2 h * q\right\} d \mathbf{x}+\int_{A_{2}} h * W d \mathbf{x} .
\end{aligned}
$$


Proof. The theorem follows from the fact that $X^{\prime}(x)=\delta X(x)$ when $\hat{E}=D$. The Corollary can be established observing that the set $\hat{E}$ satisfies the hypotheses of Lemma 1 and Eq. (40) reduces to (42) when $h \in \hat{E}$.

It must be noted that the linear space $E$ associated to $\hat{E}$ is made of those functions $h \in D_{h}$ such that

$$
h(\mathbf{x}, t)=0 \text { on } A_{1} \times\left[0, t_{1}\right] .
$$

If $E_{1}$ and $E_{2}$ are taken as the subspaces of $E$ whose elements are even and odd respectively in $\left[0, t_{1}\right]$, then:

i) $E_{1}, E_{2}$ constitute a decomposition of $E$; and

ii) The operator $L: D_{h} \rightarrow D_{h}{ }^{*}$ is positive on $E_{1}$ and negative on $E_{2}$.

Thus, according to the results of Sec. 4 , the stationary variational principle given by Corollary 8 can be transformed into a dual extremum principle.

Theorem 9. Let the set of admissible states $\hat{E}$ and the functional $X$ be given as in Corollary 8. Then

i) An admissible state $h$ is a solution of the system (22) if and only if

$$
\begin{gathered}
{\left[S_{s} \frac{\partial h}{\partial t}-\frac{\partial}{\partial x_{i}}\left(K_{i j} \frac{\partial h}{\partial x_{i}}\right)-q\right]^{\circ}=0 \text { on } R \times\left[0, t_{1}\right],} \\
h(\mathbf{x}, 0)=h_{o}(\mathbf{x}), \quad \mathbf{x} \in R, \\
{\left[W+K_{i j} \frac{\partial h}{\partial x_{i}} n_{i}\right]^{e}=0 \text { on } A_{2} \times\left[0, t_{1}\right]}
\end{gathered}
$$

and simultaneously

$$
\begin{gathered}
{\left[S_{s} \frac{\partial h}{\partial t}-\frac{\partial}{\partial x_{i}}\left(K_{i i} \frac{\partial h}{\partial x_{i}}\right)-q\right]^{\circ}=0 \text { on } R \times\left[0, t_{1}\right],} \\
h(\mathbf{x}, 0)=h_{o}(\mathbf{x}) ; \quad \mathbf{x} \in R, \\
{\left[W+K_{i i} \frac{\partial h}{\partial x_{i}} n_{i}\right]^{0}=0 \text { on } A_{2} \times\left|0, t_{1}\right| .}
\end{gathered}
$$

ii) For any pair of admissible states $h_{a}$ and $h_{b}$ that satisfy (44a) and (44b), respectively,

$$
X\left(h_{a}\right) \leq X\left(h_{b}\right)
$$

the equality holding if and only if $h_{a}=h_{b}$ is the solution of the system (22);

iii) If $h$ is the solution of the system (22), then

$\alpha)$ The maximum value of $X$ among all admissible states that satisfy (44a) is attained only at $h$;

$\beta$ ) The minimum value of $X$ among all admissible states that satisfy (44b) is attained only at $h$; and

$\gamma)$ The respective maximum and minimum values coincide.

Proof. In view of Theorem 2 and Corollary 3, and taking into account the remarks of Sec. 4 , all that is required is to verify that Eqs. $(11 \mathrm{a}, \mathrm{b})$ are equivalent to Eqs. $(44 \mathrm{a}, \mathrm{b})$, respectively. Taking the partial variations of the functional $X$ given by (42), this is verified readily.

Theorem 8 shows that for the flow problem formulated in terms of head, the difference 
$X\left(h_{b}\right)-X\left(h_{a}\right)$ can be used as an estimate of the error implied by the approximate solutions $h_{a}$ and $h_{b}$. This enhances the value of the results in applications.

iii) Formulation in terms of velocity.

Theorem 9 (Reciprocity relation). Let $\mathbf{v}$ and $\overline{\mathbf{v}}$ be two elements of the set $D_{v}$ of states defined in Sec. 6 (iii). Then

$$
\begin{aligned}
\int_{R}\left[\bar{v}_{i} * K_{i j}{ }^{-1}\right. & \left.\frac{\partial v_{i}}{\partial t}-\bar{v}_{i} * \frac{\partial}{\partial x_{i}}\left(\frac{1}{S_{\mathrm{s}}} \frac{\partial v_{i}}{\partial x_{i}}\right)+K_{i j}{ }^{-1} v_{i}(\mathbf{x}, 0) \bar{v}_{i}\left(\mathbf{x}, t_{1}\right)\right] d \mathbf{x} \\
& +\int_{A_{2}} \bar{v}_{i} n_{i} * \frac{1}{S_{\mathrm{s}}} \frac{\partial v_{i}}{\partial x_{i}} d \mathbf{x}-\int_{A_{2}} v_{i} n_{i} * \frac{1}{S_{\mathrm{s}}} \frac{\partial \bar{v}_{i}}{\partial x_{i}} d \mathbf{x} \\
= & \int_{R}\left[v_{i} * K_{i i}{ }^{-1} \frac{\partial \bar{v}_{i}}{\partial t}-v_{i} * \frac{\partial}{\partial x_{i}}\left(\frac{1}{S_{s}} \frac{\partial \bar{v}_{i}}{\partial x_{i}}\right)+K_{i j}{ }^{-1} \bar{v}_{i}(\mathbf{x}, 0) v_{i}\left(\mathbf{x}, t_{1}\right)\right] d \mathbf{x} \\
& +\int_{A_{2}} v_{i} n_{i} * \frac{1}{S_{s}} \frac{\partial \bar{v}_{i}}{\partial x_{i}} d \mathbf{x}-\int_{A_{2}} \bar{v}_{i} n_{i} * \frac{1}{S_{s}} \frac{\partial v_{j}}{\partial x_{i}} d \mathbf{x} .
\end{aligned}
$$

The functionals $\hat{L}, \hat{B}, \hat{f}$ and $\hat{f}_{B}$ corresponding to this problem may be defined in a manner similar to the previous two cases by

$$
\begin{gathered}
\langle\hat{L} \mathbf{v}, \overline{\mathbf{v}}\rangle=\int_{R}\left[\bar{v}_{i} * K_{i j}{ }^{-1} \frac{\partial v_{i}}{\partial t}-\bar{v}_{i} * \frac{\partial}{\partial x_{i}}\left(\frac{1}{S_{s}} \frac{\partial v_{j}}{\partial x_{i}}\right)\right] d \mathbf{x} \\
\langle\hat{B} \mathbf{v}, \overline{\mathbf{v}}\rangle=\int_{R} K_{i i}{ }^{-1} v_{i}(\mathbf{x}, 0) \bar{v}_{i}\left(\mathbf{x}, t_{1}\right) d \mathbf{x}+\int_{A_{1}} \bar{v}_{i} n_{i} * \frac{1}{S_{s}} \frac{\partial v_{i}}{\partial x_{i}} d \mathbf{x}-\int_{A_{z}} v_{i} n_{i} * \frac{1}{S_{s}} \frac{\partial \bar{v}_{i}}{\partial x_{i}} d \mathbf{x}
\end{gathered}
$$

which hold for every $\mathbf{v}, \overline{\mathbf{v}} \in D_{\mathbf{v}}$, and

$$
\begin{gathered}
\langle\hat{\mathrm{f}}, \overline{\mathrm{v}}\rangle=\int \bar{v}_{i} * p_{i} d \mathbf{x} \\
\left\langle\hat{\mathrm{f}}_{B}, \overline{\mathrm{v}}\right\rangle=\int_{R} K_{i j}{ }^{-1} N_{i}(\mathbf{x}) \bar{v}_{i}\left(\mathbf{x}, t_{1}\right) d \mathbf{x}+\int_{A_{1}} \bar{v}_{i} n_{i} * M d \mathbf{x}-\int_{A_{2}} W * \frac{1}{S_{s}} \frac{\partial \bar{v}_{i}}{\partial x_{i}} d \mathbf{x}
\end{gathered}
$$

For every $\overline{\mathbf{v}} \in D_{v}$, the substitution of the functional-valued operator $L=\hat{L}+\hat{B}$ and $f=\hat{f}+\hat{f}_{B}$ into Eq. (15) yields the desired formulation. Thus, an admissible function $v \in D_{v}$ will satisfy (15) if and only if it also satisfies Eqs. (23).

The corresponding stationary and dual variational principles are contained in the theorems given below.

Theorem 10. Let the set $\hat{E}$ of admissible states coincide with the whole set $D_{\text {v }}$ of states. For every state $\mathbf{v}$ of $D$ define the functional $X$ by

$$
\begin{array}{r}
X(\mathbf{v})=\frac{1}{2} \int_{R}\left\{v_{i} * K_{i j}{ }^{-1} \frac{\partial v_{i}}{\partial t}+\frac{1}{S_{s}} \frac{\partial v_{i}}{\partial x_{i}} * \frac{\partial v_{i}}{\partial x_{i}}+K_{i j}{ }^{-1} v_{i}\left(\mathbf{x}, t_{1}\right)\left[v_{i}(\mathbf{x}, 0)-2 N_{i}(\mathbf{x})\right]\right. \\
\left.-2 v_{i} * f_{i}\right\} d \mathbf{x}-\int_{A_{i}} M * v_{i} n_{i} d \mathbf{x}+\int_{A_{2}}\left[W-v_{i} n_{i}\right] * \frac{1}{S_{s}} \frac{\partial v_{i}}{\partial x_{i}} d x .
\end{array}
$$

Then

$$
\delta X(\mathbf{v})=0
$$

if and only if $\mathbf{v}$ is a solution of the flow problem specified by Eqs. (23). 
Theorem 11. Let the set $\hat{E}$ of admissible states consist of the elements of $D$ that satisfy Eq. (23d) and let $X$ be given by Eq. (51), which, with the constraint imposed on the admissible states, becomes

$$
\begin{aligned}
X(\mathbf{v})=\frac{1}{2} & \int_{R}\left\{v_{i} * K_{i i}{ }^{-1} \frac{\partial v_{i}}{\partial t}+\frac{1}{S_{s}} \frac{\partial v_{i}}{\partial x_{i}} * \frac{\partial v_{i}}{\partial x_{i}}\right. \\
& \left.+K_{i i}{ }^{-1} v_{i}\left(\mathbf{x}, t_{1}\right)\left[v_{i}(\mathbf{x}, 0)-2 N_{i}(\mathbf{x})\right]-2 v_{i} * f_{i}\right\} d \mathbf{x}-\int_{A_{i}} M * v_{i} n_{i} d \mathbf{x} .
\end{aligned}
$$

Then

i) An admissible state $\mathbf{v}$ is a solution of the flow problem described by the system (23) if and only if

$$
\begin{gathered}
{\left[K_{i j}^{-1} \frac{\partial v_{i}}{\partial t}-\frac{\partial}{\partial x_{i}}\left(\frac{1}{S_{s}} \frac{\partial v_{i}}{\partial x_{j}}\right)-f_{i}\right]^{e}=0 \text { on } R \times\left[0, t_{1}\right],} \\
v_{i}(\mathbf{x}, 0)=N_{i}(\mathbf{x}), \quad \mathbf{x} \in R, \\
{\left[\frac{1}{S_{s}} \frac{\partial v_{i}}{\partial x_{i}}-M\right]^{e}=0 \text { on } A_{1} \times\left[0, t_{1}\right]}
\end{gathered}
$$

and simultaneously

$$
\begin{gathered}
{\left[K_{i i}{ }^{-1} \frac{\partial v_{i}}{\partial t}-\frac{\partial}{\partial x_{i}}\left(\frac{1}{S_{s}} \frac{\partial v_{i}}{\partial x_{i}}\right)-f_{i}\right]^{\circ}=0 \text { on } R \times\left[0, t_{1}\right],} \\
v_{i}(\mathbf{x}, 0)=N_{i}(x), \quad \mathbf{x} \in R, \\
{\left[\frac{1}{S_{s}} \frac{\partial v_{i}}{\partial x_{i}}-M\right]^{\circ}=0 \text { on } A_{1} \times\left[0, t_{1}\right] .}
\end{gathered}
$$

ii) For any pair of admissible states $\mathbf{v}_{a}$ and $\mathbf{v}_{b}$ that satisfy (54a) and (54b), respectively,

$$
X\left(\mathbf{v}_{a}\right) \leq X\left(\mathbf{v}_{b}\right)
$$

iii) If there exists an admissible state $\mathbf{v}$ which is a solution of the system (23), then

$\alpha$ ) The maximum value of $X$ among all admissible states that satisfy (54a) is attained at $\mathbf{v}$;

$\alpha$ ) The minimum value of $X$ among all admissible states that satisfy (54b) is attained at $\mathbf{v}$; and

$\beta$ ) The respective maximum and minimum values coincide.

Proof. Notice that the set $E$ is made of the elements of $D$ that satisfy (23d) with vanishing $W$. Define $E_{1}$ and $E_{2}$ as the subsets of $E$ whose elements $\nabla$ are such that $\checkmark$ is odd and even, respectively. With these definitions $\left\{E_{1}, E_{2}\right\}$ is a decomposition of $E$. The theorem follows from the results of Sec. 4, because $L$ is non-negative in $E_{1}$ and non-positive in $E_{2}$.

\section{References}

[1] I. Javandel and P. A. Witherspoon, $A$ method of analyzing transient fluid flow in multilayered aquifers, Water Resour. Res. 5, 856-869 (1969)

[2] S. P. Neuman and P. A. Witherspoon, T'ransient flow of ground water to wells in multiple aquifer systems, Geotechnical Engineering Report 69-1, University of California, Berkeley (1969) 
[3] B. Noble and M. J. Sewell, On dual extremum principles in applied mathematics, J. Inst. Maths. Applics. 9, 123-193 (1972)

[4] B. Noble, Complementary variational principles for boundary value problems, I. Basic principles, Report No. 473, Math. Research Center, University of Wisconsin (1964)

[5] M. J. Sewell, On dual approximation principles and optimization in continuum mechanics, Phil. Trans. Roy. Soc. Lond. 265, 319-351 (1969)

[6] A. M. Arthurs, Complementary variational principles, Oxford University Press, 1970

[7] P. D. Robinson, Complementary variational principles, in L. B. Rall (ed.), Nonlinear functional analysis and applications, pp. 507-576, Academic Press, New York, 1971

[3] M. J. Sewell, The governing equations and extremum principles of elasticity and plasticity generated from a single functional. Part I, J. Struct. Mech. 2, 1-32 (1973)

[9] M. J. Sewell, The governing equations and extremum principles of elasticity and plasticity generated from a single functional. Part II, J. Struct. Mech. 2, 135-158 (1973)

[10] I. Herrera, A general formulation of variational principles, Instituto de Ingeniería, UNAM, E 10, México, D.F. (1974)

[11] M. Z. Nashed, Differentiability and related properties of nonlinear operators: some aspects of the role of differentials in nonlinear functional analysis, in L. B. Rall (ed.), Nonlinear functional analysis and applications, pp. 103-309, Academic Press, New York, 1971

[12] M. M. Vainberg, Variational methods in the study of non-linear operator equations, Holden-Day, San Francisco, 1964

[13] I. Herrera and J. Bielak, A simplified version of Gurtin's variational principles, Arch. Rat. Mech. Anal. 53, 131-149 (1974)

[14] R. S. Sandhu and K. S. Pister, Variational methods in continuous mechanics, in International conference on variational methods in engineering, Southampton University, England, 1.13-1.25 (1972)

[15] E. Tonti, $A$ systematic approach to the search for variational principle, in International conference on variational methods in engineering, Southampton University, England, 1.1-1.12 (1972)

[16] E. Tonti, On the variational formulation for linear initial value problems, Annali di Matematica pura ed applicata 95, 331-360 (1973)

[17] V. Komkov, Application of Rall's theorem to classical elastodynamics, J. Math. Anal. Applics. 14, $511-521(1966)$

[18] A. M. Arthurs, A note on Komkov's class of boundary value problems and associated variational principles, J. Math. Anal. Applics. 33, 402-407 (1971)

[19] S. P. Neuman and P. A. Witherspoon, Variational principles for confined and unconfined flow of ground water, Water Resour. Res. 5, 1376-1382 (1970)

[20] S. P. Neuman and P. A. Witherspoon, Variational principles for fluid flow in porous media, J. Eng. Mech. Div. ASCE 97, 359-374 (1971)

[21] M. E. Gurtin, Variational principles for linear initial value problems, Quart. Appl. Math. 22, 252-256 (1964) 